\title{
Cryptocurrency (Blockchain) Technology as a Means of Leveraging the Nigeria Economy
}

\author{
EBELOGU Christopher $\mathbf{U}^{1}$, ORIAKHI Joseph $\mathrm{E}^{2}$, OJO Samuel $\mathrm{D}^{3}$, AGU Edward $\mathrm{O}^{4}$ \\ Research Scholar ${ }^{1-3}$, Lecturer $^{4}$ \\ ${ }^{1-3}$ Department of Computer Science, University of Abuja, FCT Abuja, Nigeria \\ ${ }^{4}$ Department of Computer Science, Federal University Wukari, Taraba State, Nigeria
}

\begin{abstract}
Cryptocurrency is generally known to be a digital record of ownership of nominal balance which can be used to pay for transactions. Thus, for any transaction, the buyer gives instructions to transfer ownership of a certain amount of his balances to the seller. The use of cryptocurrencies such as Bitcoin and Ethereum in performing online transactions has been on the rise and almost accepted generally in the world. Africa as a continent is not left out in the adoption of blockchain and cryptocurrencies. Today, in 2019, the question of whether or not digital money can actually become a useful and secure part of the Nigerian economy is being seriously debated. This paper explores how cryptocurrency technology can be a means of leveraging the Nigeria economy and its extremely large population.
\end{abstract}

Key Words: Cryptocurrency, Blockchain, Bitcoin, Ethereum, Digital Currency, Online Transaction.

\section{INTRODUCTION}

So much has been written about cryptocurrencies and the technology involved. Still, less than $1 \%$ of the global population has ever used the digital currency (Cryptocurrency), this is according to a 2017 study conducted by Cambridge University, United Kingdom. With the slow increase rate, virtual currencies as it is alternatively called continues to make inroads into the society and has redefine the way manner we conduct and do business. The world of money and the financial ecosystem is undergoing a huge transformation before our eyes. While cryptocurrency is slowly gaining mainstream adoption, Blockchain, the underlying era at the back of cryptocurrency may offer more capacity and unlock billions of dollars in the global markets. According to a record from a research analyst on the Royal Bank of Canada, cryptocurrency and blockchain generation could unlock $\$ 10$ trillion or more in the market. A lot has been written about blockchain generation. In an easy term, blockchain is a special sort of a distributed ledger database. A disbursed ledger is sincerely a database that exists across numerous places or among more than one participant. An allotted ledger system permits company of any chain of facts or transactions without the need of intermediaries.

A cryptocurrency is a digital asset designed to work as a medium of exchange that uses strong cryptography to secure financial transactions, control the creation of additional units, and verify the transfer of assets. Cryptocurrencies use decentralized control as opposed to centralized digital currency and central banking systems. The decentralized control of each cryptocurrency works through distributed ledger technology, typically a blockchain that serves as a public financial transaction database [1]. Bitcoin, first released as open-source software in 2009, is generally considered the first decentralized cryptocurrency, since the release of bitcoin, over 6,000 alternative variants of bitcoin, or other cryptocurrencies have been created. Digital technology has become prevalent and of common use throughout the world. It is therefore expected that the mode, means, and method of performing transaction involving money needs be digitalized, therefore, necessitating the adoption and usage of digital currency [1].

\section{CRYPTOCURRENCY OVERVIEW}

The global financial system is no doubt embracing the current transition from physical currency to almost virtual currencies through the medium of technology. This wave has ushered in the birth of crypto currencies. In the light of this outbreak, there has been a lot of positive and negative discourse on the value of crypto-currencies to the Nigerian fiscal system. Investors have in their masses invested in crypto currencies, the most common being Bitcoins all in a bid to some sort of recoup interests in the nearest future. Over time the awareness that bitcoins like most crypto currencies operates independently and outside the control or 
regulation of any intermediaries such as banks, financial institutions, or the government triggered a wakeup call directed to the risks investors may be exposed to when involved in this venture.

Economics research to this point has furnished little perception into the economic relevance of cryptocurrencies. Most current model of cryptocurrencies are built by Computer scientists who mainly focus at the feasibility and security of those systems. Crucial troubles coupled with the incentives of contributors to cheat and the endogenous nature of a few key variables along with the real value of a cryptocurrency in exchange have been in large part ignored. Such considerations, however, are pivotal for understanding the optimal layout and, hence, the financial price of cryptocurrency as a means of payment.

Relatively, the Nigeria government has attempted to place a ban on cryptocurrency, although its legal status remains ambiguous unlike in countries like Morocco and Algeria where there is a clear ban on trading in Bitcoins such that a breach attracts heavy fines.

Currency as a legal tender is accepted as a unit of account, store of value and medium of exchange [2], money originated through public enterprises in the ancient Mesopatamian temples and palaces as standardized weight and also assisted in the development of internal accounting for recognition of credits and debits and as an instrument of taxation [3] \& [4]. According to [5] \& [6], money predated minting for about 3000 years. Considering the development of money in Egypt, it became clear that the State plays important role in establishing the appropriate measure of value for the purpose of accountability [6]. Cowries, Fijian whales' teeth, Yap stones, Wampum, cattle and metallic currencies were the earliest forms of money in existence and thereafter, notes were issued as money but in each instance, the State played important roles such as determining the value of the currency and administrative control [4], [5], [6].

However, the arrival of cryptocurrency which has its root in cryptographic technology with online distributed ledger appears to be introducing the finance industry into another phase (fourth) of development which incredibly is a combination of both the Central Bank function as well as Accounting functions as it produces and manages the supply of virtual currency as well as producing and managing the record of transactions simultaneously [7]. This technology is indeed a pointer to the fact that we have moved to the era of the fourth industrial revolution.

\subsection{Anonymity}

The concept of anonymity implies that transacting parties do not know the real identity of each other. This feature of anonymity most times is reckoned as strength of the Bitcoin technology. Anonymity complicates the possibility of identifying individuals who engage in illegal transactions and other illicit activities [8]. However, individuals with a single wallet address for various transactions stand the risk of having their details tracked down by smart attacker who can swiftly identify the user's alphanumeric keys. It could be said that the participants duly recognize the risks and are willing (by their signature) to totally lose out in the event of sudden attack. In the occasion of death of a crypto account owner, his wealth automatically goes into extinction. Whereas, in the traditional banking system, allowance exists for next of kin to claim the benefits of the deceased because anonymity implies that no other person except the owner of the crypto account could access and extract his details from the account data. With the knowledge of the risks, the participants who invest heavily and frequently could be suspected to be potential attackers or those who engage in illicit transactions, ceteris paribus [9]. They invest either to attack or to be attacked. Further research can be carried out on this. Although, the regime of Know Your Customer (KYC) could help, [10] posits that it weakens anonymity which innocent users might seek to enjoy.

\subsection{Lower Transaction Cost}

Operating cryptocurrency account is believed to be cheap because the services of experts like bankers who act as intermediaries, are not required to verify transactions as in the traditional business [11]. Although, transactional costs may appear low but in actual fact, the miners are paid substantial amount for the process of mining. Again, it has been argued that the lower cost of transactions may not be sustainable in the future.

\subsection{International Acceptance}

This attribute of Bitcoin stemmed from the fact that Bitcoin could promote a cashless environment and overcome all the limitations posed by the use of cash. Being an online-based transaction which could be easily accessed by anyone, this innovation promises to be less strenuous, allowing any unit of transaction, with no territorial boundary. European Union (2012) viewed a unit of Bitcoin as being divisible to eight decimal places and can be used in any kind of transaction irrespective of the magnitude. This is where Bitcoin differs again from other forms of currencies. These features present cryptocurrency as very attractive to users. However, IMF (2016) refuted the claim that Bitcoin enjoys universal acceptance as a medium of exchange. This is because, certain nations have publicly declared the use of cryptocurrency illegal and would be ready to prosecute anyone that contravenes 
such policy (The Law Library of Congress, 2018). Other features "cum benefits" that could make the use of cryptocurrency attractive includes foreign payments and payments in countries with high inflation rate [12]. Even, though it seems to gain attraction from many users, no country had legislated its use thus far.

\subsection{Lack of Central Authority}

Lack of central authority is an in-built feature of Bitcoin Technology which connotes that there is no single administrator or an entity particularly in charge of the administration of cryptocurrency. All users are independent of one another. Although, the account details of transactions of each participant are within the public domain, yet, there is no responsible authority for the coordination of its activities. Cryptocurrency accounts maintenance contains an inbuilt, self-regulating mechanism that is similar to internal control mechanism found in client-oriented organizations. Transactions are chronologically dependent on each other by virtue of the cryptographic hash of the preceding block contained in the subsequent block. It was launched by anonymous developer(s) and patronized by anonymous users FATF in 2016. [8] \& [9] noted the difficulty of identifying, locating and holding any individual or entity liable by law enforcement agents for investigative purpose since there is no traceable central administrator in charge of the functionality of Bitcoin. This feature of Bitcoin appears very risky for investors in case of any unforeseen contingencies. This accounts for one of the main reason why no country is eager to legislate its use [13]. The problem of hoarding of currency by the wealthy few for speculative reason could also throw the economy into a serious mess [2]. This problem can become worse, in a case which is similar to Bitcoin where there is no central authority.

\section{CRYPTOCURRENCY IN NIGERIA}

Considering the experience of Nigeria, Ponzi schemes appeared to have gained popularity among the Nigerian citizens prior the introduction of Bitcoin. A Ponzi scheme according to the U.S. SEC (2018) is "an investment fraud that involves the payment of purported returns to existing investors from funds contributed by new investors". Ponzi schemes have been described as financial frauds under the promise of high profits by [14]. [15] Conducted an empirical study on Bitcoin-based scams and reported that $21 \%$ of 192 cases of Ponzi schemes in Nigeria, Forex Trading, Scam Wallets, Mining Scams, and some other fraudulent exchanges were all associated with Bitcoin addresses. Likewise, [16] provided the cases of theft involving Bitcoin during years 2013 to 2015 with the names the companies affected and the amount lost. During year 2017, the Federal Government has made official release twice, intimating citizens about its non-readiness to support the use of Bitcoin and warning individuals, corporate bodies and Banks not to get involved in Bitcoin transactions. This warning became imperative because of the devastating effects of the various Ponzi schemes in Nigeria which suddenly collapsed towards the end of year 2016 up to the first quarter of 2017 in which case, many Nigerians lost their fortunes [14]. This study was undertaken to ascertain the view of Nigerian Professional Accountants towards legislating cryptocurrency in Nigeria. It also examined the ostensible benefits from the legislation of cryptocurrency. It was hinged on Chartalism theory which states that the origin of money is located outside private markets and rests within the complex web of social (debt) relations where the state has a principal role. This implies that the state both denominates and institutes the role of money (currency) as a unit of account prior its role as a means of payment and a medium of exchange. Only the government has the power to levy taxes and to declare what will be accepted at pay offices for extinguishing debt to the state. Ingham (2000) maintained that the essence of state as the final authority does not lie in the ability to create laws or to print money, but in the ability of the government to create 'the promise of last resort. With the introduction of cryptocurrency, the Central Bank of Nigeria must still be in place to guarantee monetary stability and political legitimacy with the introduction of the new electronic form of legal tender (cryptocurrency) with regard to the soundness of monetary hierarchy of the issuing institution or state [17].

The crypto currency market has grown over the years and it keeps expanding as new crypto currencies emerge more frequently. As at August 2018, records revealed the existence of over 1,600 cryptocurrencies available on the internet. Currently, there are over 2000 crypto currency and virtual currencies in the world, this goes to show the speed at which it grows and expands.

Some major types of cryptocurrencies presently in use in Nigeria include Bitcoins, Litecoin, Zcash, Dash, Ripple, Ethereum, NEO, Altcoins and Tether. Bitcoin is widely seen as a pioneer and the most successfully used in the world of crypto currencies. Bitcoin was first made available to the public in 2009, and has ever since expanded by maintaining the highest market capitalization.

\section{Major features associated to cryptocurrencies include:}

$>$ Created to be Anonymous: As the name implies, crypto means something concealed in secrecy or hidden. Their operation involves the use of codes in a form known as cryptography. 
$>$ Designed to operate with limited control from the government: Crypto currencies are modeled to operate with absolute independence. No central authority, government or cooperation has access to the funds or personal information of the currency owners.

$>$ Funds transfer is carried out directly between parties, and in the absence of any third party like a bank or credit card company.

In a nutshell, the state determines what to accept and what not to accept as a medium of exchange. Therefore, the power to either abate or legislate the use of cryptocurrency by citizens of any country resides with the state.

\subsection{Amazing Potentials Using the Cryptocurrency Technology in Nigeria}

Despite its little setbacks, Cryptocurrency ought to adversely transform the current economic state of affairs in Nigeria, absolutely starting with the convenient cross-border transfers and ending with the payment of employees' salaries in cryptocurrency. However, current blockchain platforms are limited by the boundaries of their very own ecosystems and that they do no longer interact with each other accordingly, losing the ability for global scalability. Blockchain era and the virtual currencies created on its foundation can exponentially lessen the price of global transactions, along with making those transactions genuinely free. Trade, at all levels, has continually been focused on uncertainty and trust. Banks, corporations, governments, or even universities are merchandise of uncertainty and lack of trust between transacting partners. These centralized establishments have become the grease that stored the monetary wheel turning. However, the institutions have become all too powerful and are actually working in the direction of consolidating their power. Though blockchain generation was simplest added in 2009, it remains a novel manner of decreasing uncertainty among transacting parties.

Cryptocurrency exchanges cannot be ignored, as they may be the institutions that maintain the whole enterprise going whilst people change the virtual coins. Programmers, scriptwriters, journalists, picture designers, marketers, and many more have carved a success careers out of blockchain and cryptocurrencies innovation.

Digital currencies are gradually converting how wealth is created and distributed. A level playing field has been created and everybody has an equal possibility to make money. An authentic egalitarian financial society is in motion; however a few governments are combating cryptocurrencies via innovation-inhibiting regulations.

\section{PRACTICAL APPLICATIONS OF BLOCKCHAIN IN NIGERIA}

Blocks at the blockchain store records economic transactions; we now have successfully gotten that out of the settled. But blockchain is clearly a much more reliable way of storing records about other types of transactions, as well. In fact, blockchain technology may be used to store records related to properties, monitor fluctuations in the supply, or even votes for a candidate or a general election.

Deloitte (A Professional services network) lately surveyed about 1,000 agencies across seven countries requesting opinions on integrating blockchain into their business operations. Their survey found that $34 \%$ already had a blockchain operations in manufacturing today, another $41 \%$ already have the plan of installing the blockchain application in the space of 12 months. In addition, almost $40 \%$ of the surveyed businesses reported they would invest $\$ 5$ million or greater in blockchain coming year. Here are a number of the most popular applications of the blockchain technology being explored today.

\subsection{Bank Usage}

Obviously no business setup stands to gain from integrating blockchain in operations other than banking. Financial institutions have limited hours used for business operations within the 5 days in a week (Mondays to Fridays). That means in case you try to deposit a check on Friday at 6 p.m., you in all likelihood have to wait until Monday morning to see that cash hit your account. Even in case you make your deposit within the normal working hour, the transaction can nevertheless take between 1 - 3 days to have your transaction (s) duly processed simply because of high volume of transactions banks do have to process but this can never be the case if Blockchain technology is used in this regard. In a lighter note, blockchain never goes to bed.

By integrating blockchain into the banking sector, customers can see their transactions processed in as fast as 10 minutes, essentially the time it takes to add a block to the blockchain, no matter the time or day of the week. With the injection of the blockchain technology, banks also have the opportunity to move funds between from one bank or establishments to another in a far greater, quicker and more secure way. In the inventory buying and selling enterprise, for example, the settlement and clearing procedure can take up to a few days (or longer, if banks are trading internationally), which means that the money and stocks are 
frozen for that time. Given the dimensions of the sums involved, even the few days that the cash is in transit can deliver significant expenses and risks for banks.

Also, the customers/users of this technology have no fear of bank collapsing due to financial irregularities and misappropriation of funds by the different CEOs and Managing Directors as it has been seen for some decades in the Nigerian banking system. Customers can easily transfer all funds from their collapsing bank if this is noticed to their other blockchain accounts instantly.

\subsection{Use in Cryptocurrency (Bitcoin and other Digital Currencies)}

Blockchain forms the bedrock for cryptocurrencies like Bitcoin. As we earlier explored, currencies like the United States Dollars (USD) are regulated and verified by a centralized authority, normally a financial institution or government. Under the centralized system, user's information and finances are technically on the whim of their financial institution or government. If the financial authorities (Banks) collapse or they stay in a country with an unstable government, the price of their foreign money can be at risk. These are the concerns out of which Bitcoin came into recognition.

By spreading its operations across a network of computers, blockchain allows Bitcoin and different digital currencies to operate with no need for any central controlling authority. This reduces risk and also eliminates many of the processing and transaction fees. It additionally gives those with volatile currencies a more stable Forex with more packages and a wider community of people and institutions they are able to do business with, both regionally and internationally (at least, this is the goal.)

\subsection{Healthcare Usage}

The health sector can leverage on the blockchain technology to securely store patients' medical data anytime of the day. When a medical record is generated and signed, it may be written into the blockchain, which gives the patients the proof and confidence that the file cannot be changed. These personal health information/data/records may be encoded and saved at the blockchain with a non-public key, so they are most effective reachable by positive individuals, thereby making sure privacy.

\subsection{Property Records Usage}

If you have ever had some moments of interactions with your local Recorder's Office, you will recognize that the technique of recording property rights in Nigeria is mostly cumbersome and inefficient. Today, a physical deed has to be brought to a central authority employee at the closest recording office, from where it will be manually entered into the Nation's centralized database and public index. In the case of an assets dispute, claims to the belongings need to be reconciled with the public index. This process is not just high priced and time-consuming; it's also riddled with human error, where every inaccuracy makes monitoring of property ownerships ineffective. Blockchain has the ability to take away the need for scanning documents and tracking down files in a recording office. If properties and its ownership records are stored and verified on the blockchain, owners can consider that their deed is accurate and permanent.

\subsection{Use in Smart Contracts}

Smart Contracts refer to computer codes that can be constructed into the blockchain to facilitate, verify, or negotiate agreement of contracts. Smart contracts operate under some conditions that customers agree to. When those situations are met, the terms of the settlement are routinely carried out.

We assume that, I'm renting you my lodge using a Smart Contracts. I agree to present you the door code to the lodge as soon as you pay me your security deposit, both parties would forward portion of the deal to the smart contracts, which is to be observed. If I don't deliver the door code through by the agreed rental date, the smart contracts refund your safety deposit. This gets rid of the prices that usually accompany using a notary or any outside mediator (that is, the normal organization fee or agent that we are used to in Nigeria); this applies also to Lands, Houses etc. With the introduction of the technology, all of those might be removed and transparent change of contracts will come to play.

\subsection{Supply Chain Use}

Suppliers can also leverage on the blockchain technology to record the origins of materials that they have purchased. This might allow agencies to verify the products' authenticity, along with health and ethics labels like "Organic," "Local," and "Fair Trade." Based on Forbes' reports, the food enterprise is getting into using blockchain to increasingly monitor the path and safety of food throughout the farm-to-user journey. 


\subsection{Election Usage (Voting)}

Voting with blockchain incorporates the ability to put off election fraud and enhance voter turnout, as become tested inside the November 2018 midterm elections in West Virginia, United State of America. Each vote could be saved as a block on the blockchain, making them nearly not possible to tamper with. The blockchain protocol could also hold transparency in the electoral process, reducing the personnel to conduct an election and offer officials with immediately results. The post and aftermath of election violence could be reduced drastically, if not definitely wiped off with this technology.

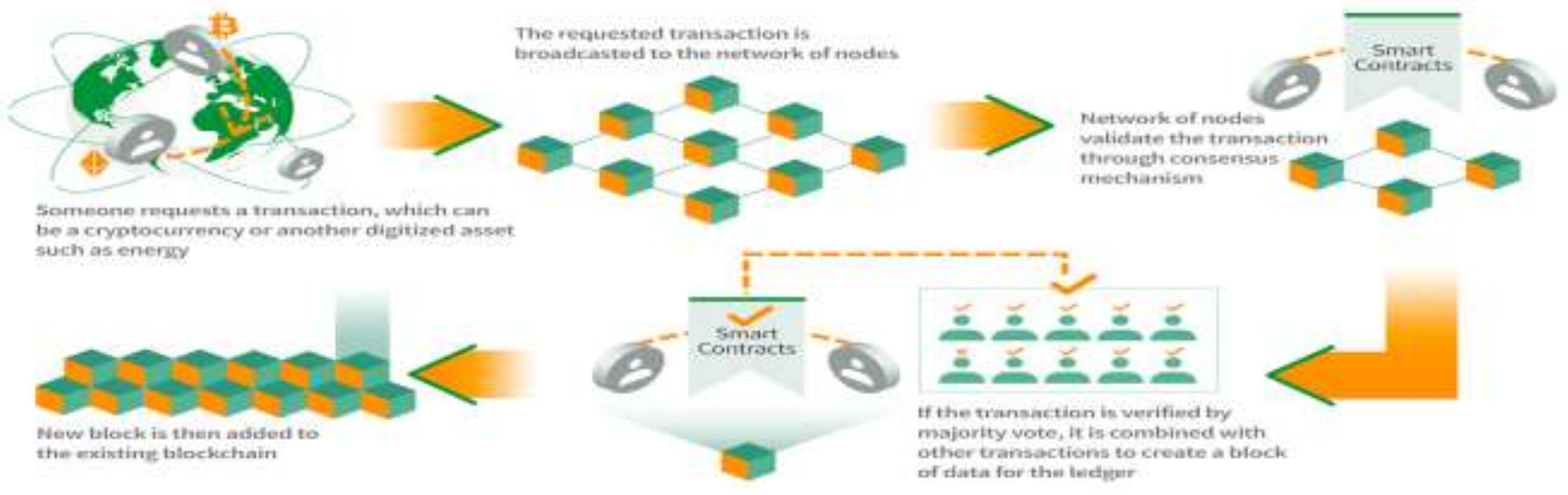

Fig 1: Cryptocurrency and the Digital Transaction Process

\section{BLOCKCHAIN TECHNOLOGY HIGHPOINTS}

For all its complexity, blockchain's potential as a decentralized mode of record-keeping is almost without limit. From greater individual privacy and heightened protection to lower processing charges and fewer errors, blockchain era may very well see applications beyond the ones outlined above.

- Improved accuracy by disposing of human involvement in verification.

- Cost elimination by doing away with third-party engagement and verification.

- Decentralization makes it tougher to tamper with.

- Transactions are secured, private and very efficient.

- Transparent technology

Below are the beauties of the blockchain technology for businesses/companies/firms available on the market today.

\subsection{Improved Accuracy}

Transactions at the blockchain network are approved through a community of heaps or thousands and thousands of computers. This removes almost all human involvement inside the verification process, ensuring in much less human error and a more accurate record of information. Even if a laptop makes a computational mistake, the error would be limited to only one line of blockchain. In order for that errors to go to the rest of the blockchain, it would want to be made by at least $51 \%$ of the community's computers; a near impossibility.

\subsection{Cost Elimination}

Basically, many clients pay a financial institution to verify a transaction, a notary to sign a document, or a minister to carry out a marriage. Blockchain removes the necessity for third-party verification and, with it, their associated costs. Businesses and agencies incur a small fee each time they accept bills using credit-cards, for example, due to the fact that banks must process the transactions. Bitcoin, on the other hand, does not have a central authority hence no charges or costs related to the transaction.

\subsection{Decentralization (making it very tough to tamper with)}

Blockchain does now not store any of its data/records in a centralized location. Instead, the blockchain is copied and spread throughout a network of computers. Whenever a new block is brought to the blockchain, every computer on the community updates its blockchain to reflect the change. By spreading that statistics across a network, rather than storing it in a single 
database, blockchain becomes tougher to tamper with. If a duplicate of the blockchain fell into the hands of a hacker, simplest a single reproduction of the information, rather than the entire network, would be compromised.

\subsection{Efficient Transactions}

Transactions carried out through a central authority can take days to materialized/processed. If you decide to deposit a check on Friday night for example, you could not really see funds for your account till Monday morning. Whereas banks or other monetary establishments operate for the duration of stipulated hours, 5 days a week, blockchain on the other hand operates 24 hours a day, seven days a week. Transactions may be finished within ten minutes and be seen secured in few hours. This is particularly essential for cross-border trades, which commonly take longer time due to time-zone issues and for the fact that all those involved must verify processing of the payment.

\subsection{Private Transactions}

So many blockchain networks operate as public databases, which imply that absolutely everyone with an internet connection can view a list of the network's transaction history. Although users can get additional information about transactions, they cannot notwithstanding have an access to seeing detailed information about the users of such information. It is a common misperception that blockchain networks like bitcoin are anonymous, whilst in reality they are not confidential. That is, when transactions are made public by the user, their particular code called a public key, is recorded on the blockchain, rather than their personal data. Although a person's identity continues to be related to their blockchain address, this prevents hackers from acquiring a consumer's personal facts, as it is in the case of other physical financial institutions.

\subsection{Secured Transactions}

Immediately a transaction is recorded, its authenticity has to be verified by the blockchain network. Millions of computer systems at the blockchain rush to affirm that the information of the purchase is correct. After a computer has verified the transaction, it gets included on the blockchain in the like a block. Each block on the blockchain incorporates its own specific hash, along with the unique hash of the block earlier than it. When the facts on a block is edited in any way, that block's hash code changeshowever, the hash code at the block after will not. This discrepancy makes it extremely hard for records on the blockchain to be modified without notice.

\subsection{Transparency}

Although, individuals' records as domiciled on the blockchain network are secured, the technology on its own context is a public one. This simply means, that users on the blockchain network can regulate the code as they see fit, so long as they have got a majority of the network's computational authorities backing them. Keeping records on the blockchain additionally makes tampering with data a whole lot more difficult. With millions of computers on the blockchain network at any given time, for example, it is unlikely that all and sundry could make a change when no one noticed.

\section{THE FUTURE}

So many have seen cryptocurrencies as a solution that might bring to an end the fiat currencies; this is far from being true. Cryptocurrency and blockchain innovations have a long way to go before they can completely extinguish the existing financial structure. While the global acceptability of cryptocurrencies has steadily increased, nevertheless, it is still obviously in the minority. For this to happen there is a need for vast acceptance amongst consumers. However, their relative complexity compared to conventional currencies will likely discourage so many people, except for the technologically adept.

It would additional be naïve to assume that blockchain technology can solve the entire world's financially transactions or problems. It is nevertheless a long way from doing so. It is young, and requires more research and mistakes must be made before the innovation can attain its complete potential. However, we cannot take away how it has impacted the economic and financial gadgets in the world. Cryptocurrency can assist the global economy, and the reality that Wall Street frets about it is a signal that these alternative digital currencies are here to stay.

\section{CONCLUSION}

Digital currencies were virtually and largely absent in the economic scene during the 20th century. They are a relatively new invention and have remained with us for a while. The acceptability and usage of digital currencies is hinged on individuals and countries perspective. For instance, a country like China has explicitly or implicitly bans them while some other country, like Switzerland, are striving so hard and making frantic efforts to attract digital currency traders, investors and operators. 
Conventional money is also being substituted with digital currencies as observed recently in Venezuela. Venezuela plans to issue her own national digital currencies (The Economist, 2018). In most developed countries, especially in major jurisdictions, authorities have adopted the "wait and see" attitude, while they closely monitor developments in the digital currencies technology. Blockchain technology has its own fair share of advantages that go beyond the financial sector (protected assemblage of important data and information, such as scientific bills, health records, vote records etc.). And with all these said, the worldwide influence of the blockchain and cryptocurrency is anticipated to increase and also become even more firmly established and robust.

Although, several financial regulatory authorities and institutions issued casual warnings to the general public, advising of the risks of involvement in digital currencies; however, digital currencies (especially cryptocurrencies) are thriving since the number of businesses and persons who accept them as payment are increasing by the day. A significant number of people are now fully convinced that the digital currency-Bitcoin is legitimate, safe and has value. "Bitcoin is going to be transformative" but unfortunately, since it is virtually untraceable it has been used for a host of nefarious purposes. Although, digital currencies may be very attractive to cybercriminals and fraudsters and present a host of new challenges to law enforcement in Nigeria, its adoption as a tool for national development in the digital age should be widely encouraged. According to forecasts from Statistic, by 2021 the size of the blockchain technology market will grow to over $\$ 2$ billion.

\section{REFERENCES}

1. Johnson F, Akande A., Akinsanya P. (2019). Leveraging digital currency For National Development. Retrieved from https://www.academia.edu/38942203/LEVERAGING_DIGITAL_CURRENCY_FOR_NATIONAL_DEVELOPMENT

2. Gaudamuz, A., \& Marden, C. (2015). Blockchains and Bitcoin: Regulatory responses to Cryptocurrencies. First Monday, 20(2).

3. Forstater, M. (2005). Taxation and primitive accumulation: The case of colonial Africa. In Tcherneva, P.R. (eds), The Nature, Origins, and Role of Money: Broad and Specific Propositions and Their Implications for Policy. Working Paper No. 46.

4. Tcherneva, P.R. (2005). The nature, origins, and role of money: Broad and specific propositions and their implications for policy. Working Paper No. 46.

5. Davies, G. (2002). A history of money from ancient times to the present day, (Third Edition). University Of Wales Press Cardiff.

6. Henry, J.F. (2004). The social origins of money: The case of Egypt. European Journal of Political Economy, 14, 407-432.

7. Zheng, Z., Xie, S., Dai, H., Chen, X., \& Wang, H. (2017). An overview of blockchain technology: Architecture, consensus, and future trends. IEEE 6th International Congress on Big Data, 557-564.

8. Zamani, E.D., \& Babatsikos, I. (2017). The use of Bitcoins in light of the financial crisis: The case of Greece. The 11th Mediterranean Conference on Information Systems (MCIS), Genoa, Italy.

9. Meiklejohn, S., Pomarole, M., Jordan, G., Levchenko, K., McCoy, D., Voelker, G.M., \& Savage, S. (2016). A fistful of Bitcoins: Characterizing payments among men with no names. Communications of the ACM, 59(4), 86-93.

10. Carlisle, D. (2017). Virtual currencies and financial crime challenges and opportunities. RUSI Occasional Paper.

11. Dierksmeier, C., \& Seele, P. (2016). Cryptocurrencies and business ethics. Journal of Business Ethics, 1-14.

12. He, D. (2018). Monetary policy in the digital age: Crypto assets may one day reduce demand for central bank money. $A$ Quarterly Publication of the International Monetary Fund, 55(2), 20-21.

13. Godlove, J.D.N. (2014). Regulatory overview of virtual currency. Oklahoma Journal of Law and Technology, $10(1), 1-67$.

14. Bartoletti, M., Carta, S., Cimoli, T., \& Saia, R. (2017). Dissecting Ponzi schemes on Ethereum: Identification, analysis, and impact. Retrived from: https://arxiv.org

15. Vasek, M., \& Moore T. (2015). There's no free lunch, even using Bitcoin: Tracking the popularity and profits of virtual currency scams. International Conference on Financial Cryptography and Data Security. http://citeseerx.ist.psu.edu/viewdoc/download?doi=10.1.1.671.1283\&rep=rep1\&type=pdf

16. Gilbert, S., \& Loi, H. (2018). Digital currency risk. International Journal of Economics and Finance, 10(2), 108-123.

17. Miller, R. Michalski, W., \& Stevens, B. (2002). The future of money. Organisation for Economic Co-Operation and Development. OECD Publication. 